\title{
Noise influence on the Characteristic Relations and Reinjection Probability Densities of type-II and Type-III Intermittencies
}

\author{
Ezequiel Del Rio ${ }^{1}$, Sergio Elaskar ${ }^{2}, J_{. M}$ Donoso $^{1}$ and L. Conde ${ }^{1}$ \\ ${ }^{1}$ Dept. Física Aplicada. ETS Ingenieros Aeronáuticos \\ Univ. Politécnica de Madrid. 28040 Madrid. Spain \\ Email: ezequiel.delrio@upm.es \\ ${ }^{2}$ Dept. Aeronáutica. Facultad de Ciencias Exactas, Físicas y Naturales. \\ Univ. Nacional de Córdoba. Avda. Vélez Sarfield 1611. \\ Córdoba 5000. Argentina. \\ Email: elaskar@efn.uncor.edu
}

\begin{abstract}
This paper explores the effect of the noise in the reinjection probability densities (RPD) for type-II and type-III intermittencies by using the temporal series of iterative maps. The RPD are calculated by means of a new method proposed in Refs. [1] and [2] and the results are compared with both, numerical simulations and analytical calculations. In addition, we provide an explanation for the gap observed in early experiments around the unstable point in the Poincaré map. We show that and added white noise approaches the RPD to the case of uniform reinjection for small distances of iterations to the unstable point. For large distances the RPD should be incremented with respect to the noiseless case. These numerical results suggest the existence of a noise induced reinjection mechanism. density

Keywords: Intermittency. Characteristic relations. Reinjection probability
\end{abstract}

\section{Introduction}

The intermittency is a particular form of deterministic chaos where transitions between different behaviours of the system take place. The system exhibits a regular response until a small change in a given parameter brings it into a different regime. This results into a time dependent response where chaotic bursts are separated at regular intervals by a laminar or regular responses [3]. The original idea was introduced in connection with the Lorentz system $[4,5]$ but the intermittent behaviour is widespread in nature. It has been observed in the Bénard convection experiments [7], nonlinear electronic circuits $[2,8,9,10]$ or in the human heartbeats time series [11].

In the type-II and III intermittencies, the reinjection mechanism from the chaotic motion into the laminar region depends on the dinamic of the chaotic phase. This constitutes global property and it was pointed out as a relevant factor in the scaling time duration of the laminar region [8, 10, 12]. This reinjection process from the chaotic response back into the regular or laminar 
Del Río, E. et al.

response could be characterized by means of a reinjection probability density (RPD). However, only in few cases it is possible to derive an analytical expression for the RPD $\phi(x)$ or to obtain it numerically or from experimental data or time series. The reason is the huge number of sampling data required to cover each small interval of initial conditions located within the reinjection zone.

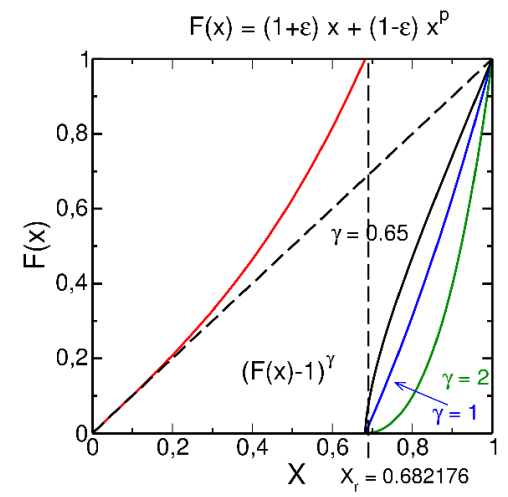

Figure 1. The map of Eq. (1) with $p=3$ and $\varepsilon=10^{-3}$.

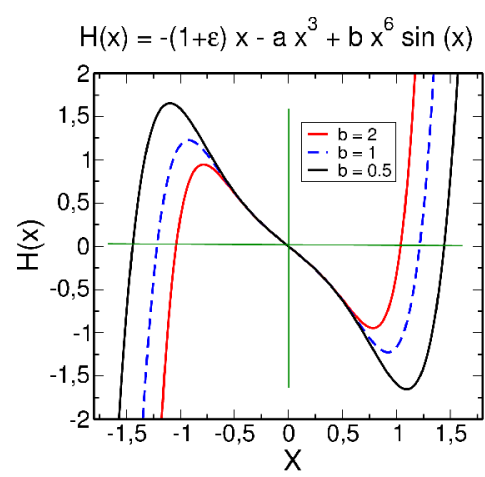

Figure 2. The map of Eq. (2) for $a=1$ and $\varepsilon=10^{-3}$.

In consequence, different simplifying assumptions have been proposed for $\phi(x)$. The widespread approximation considers a uniform RPD independent of the reinjection point $[3,4,7-10,13-16]$ or a rather artificial assumption considers that the reinjection occurs at a fixed point $[10,15]$.

In this note we make use of a new approach to derive the RPD from numerical data of time series obtained from iterative maps. The RPD is calculated using iterative data series of one dimensional maps which exhibit type-II and type-II intermittencies.

\section{Model maps}

In order to model the type-II intermittency we analyze the following one dimensional map,

$$
x_{n+1}= \begin{cases}F\left(x_{n}\right) & x_{n} \leqslant x_{r} \\ \left(F\left(x_{n}\right)-1\right)^{\gamma} & x_{n} \geqslant x_{r}\end{cases}
$$

where $F\left(x_{n}\right)=(1+\varepsilon) x_{n}+(1-\varepsilon) x_{n}^{p}$ and $x_{r}$ is the root of $F\left(x_{r}\right)=1$ as it could be observed in Fig. 1. The type-III intermittency is modelled by using the map $x_{n+1}=H\left(x_{n}\right)$ where the function,

$$
H\left(x_{n}\right)=-(1+\varepsilon) x_{n}-a x_{n}^{3}+b x_{n}^{6} \sin \left(x_{n}\right)
$$

is represented in Fig. 2 and is usually approximated by,

$$
H\left(x_{n}\right) \approx-(1+\varepsilon) x_{n}-a x_{n}^{3}
$$

and this last expression corresponds to the local Poincare map for type-III 
intermittency.

In Fig. 1 the origin $\quad x=0$ is always an stable fixed point for $\varepsilon<0$ that becomes unstable for $\varepsilon>0$. The successive iterations from an initial value

$x_{o}$ close to the origin, bring $x_{n}$ away in a process driven by the parameters $\varepsilon$ and $p$. When $x_{n}$ becomes larger than $x_{r}$, a chaotic burst occurs, that will be interrupted when $x_{n}$ is again mapped back into the laminar region of low values of $x_{n}$.

For $\gamma=1$ for the map of Eq. (1) we have $x_{n+1}=F\left(x_{n}\right) \quad(\bmod 1)$ while for $p=2$ we recover the map used by Manneville in his pioneer paper [4]. The case $p=3$ corresponds with the local Poincare map of type-II intermittency for points close to $x=0$. Both parameters $\varepsilon$ and $p$ modify the duration of the so called laminar phase, where the system exhibits a quasiperiodic or regular response.

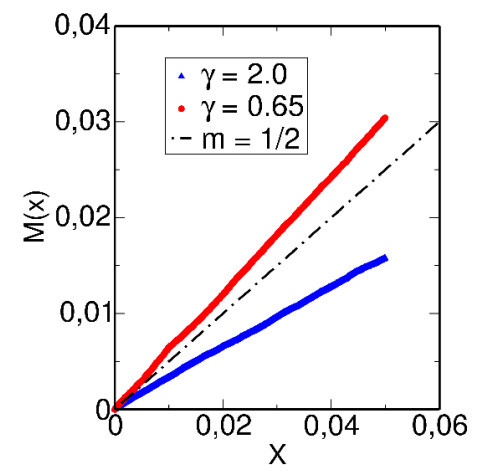

Figure 3. The values of $M(x)$ (solid points) for the map of Eq. (1) for two values of $\gamma$

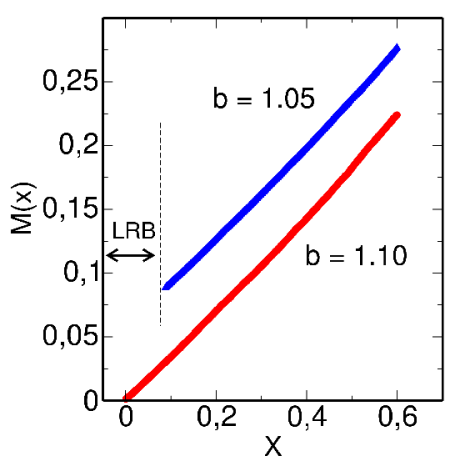

Figure 4. The function $M(x)$ (solid points) for the Eq. (2) for two values $b$ showing $x_{i}>0$.

The RPD is strongly dependent on the parameter $\gamma$ which, as it could be observed in Fig. 1, determines the curvature of the map in region past $x_{r}$ Then, only points $x>x_{r}$ are iterated back into of the laminar region around the unstable fixed point at the origin. As it could be observed in Fig. 1 larger values of $\gamma$ increases the curvature of the function and, consequently, a larger fraction of iterated points are therefore mapped close to the unstable fixed point at $x=0$.

\section{Reinjection Probability Distribution}

It is difficult to derive an analytical expression for $\phi(x)$ because it relies on the chaotic response of the system. In order to obtain a semiempirical expression for the RPD, we make use the function $M(x)$ defined in Refs. [1] and [2], 
Del Río, E. et al.

$$
M(x)=\frac{\int_{o}^{x} s \phi(s) d s}{\int_{0}^{x} \phi(s) d s} \text { if } \quad \int_{0}^{x} \phi(s) d s \neq 0
$$

and $M(x)=0 \quad$ otherwise. This average allows a more reliable experimental and numerical access than $\phi(x)$. The evaluation of $M(x)$ for the maps of Eqs. (1) and (2) are shown in Figs. 3 and 4 where the case of uniform reinjection corresponds to the dot dashed line with slope $\phi(x)$.

The numerical values of $M(x)$ could be approximated by a linear function $M(x)=m\left(x-x_{i}\right)+d$, where $x_{i}>$. is the low boundary reinjection (LBR) point. This latter is defined as the lower value of $x$ where a reinjection event occurs and is also indicated in Fig. (4). In addition, $x_{c}>x_{i}$ represents the maximum value where a laminar or regular response is observed. Assuming a linear expression for $M(x)$ we can solve the Eq. (3) and we have,

$$
\phi(x)=b\left(x-x_{i}\right)^{\alpha} \quad \text { with } \quad \alpha=\frac{1-r m}{m-1}
$$

The constant $b$ is determined by the by the normalization condition,

$$
N \int_{x_{i}}^{x_{c}} b\left(x-x_{i}\right)^{\alpha} d x=1
$$

The RPD must be symmetric in the map of Eq. (2), and the factor $N=2$ in Eq.(6) is introduced to account for the negative $(x<0)$ reinjections, while for Eq. (1) we have $N=1$. When $\alpha>-1$, or equivalently, for $0<m<-1$, the above integral gives,

$$
b=\frac{1}{N} \frac{\alpha+1}{\left(x_{c}-x_{i}\right)^{\alpha+1}}=\frac{1}{N} \frac{m}{1-m}\left(x_{c}-x_{i}\right)^{m /(m-1)}
$$

which permits to evaluate the RPD. Therefore, the value of $m$ obtained from the numerical calculations of Figs. 2 and 3 determines the RPD using the Eqs. (5) and (6). Then, the approximation $M(x)=m\left(x-x_{i}\right)+d$, determines $\phi(x)$ which only rely the density is determined only by the parameters $m$ and $d$ that are easier to calculate than $\phi(x)$.

The RPD obtained using the linear approximation for Eq. (3) are in agreement with the numerical calculations of $\phi(x)$ as evidenced in Figs. 2 and 3. The RPD obtained in the numerical calculations using a huge number of iterations of both maps are represented together with the functions $\phi(x)$ given by Eq. (4) obtained by the linear fits of $M(x)$ in Figs. (2) and (3).

From Eq. (5), is also deduced that the properties of the RPD close to $x=x_{i}$ differ accordingly to the value of $m$. That is, we have $\lim _{x \rightarrow x_{i}} \phi(x)=\infty$ when $0<m<1 / 2$ while for $1 / 2<m<1$ we obtain $\lim _{x \rightarrow x_{i}} \phi(x)=0$ and the value $m=1 / 2$ corresponds to the uniform RPD $\phi(x)=1 / x_{c}$. Therefore, from Eq. (3), we expect for $\gamma>1$ values of $m$ in the interval $0<m<1 / 2$ and for $\gamma<1$ values in the interval 
$1 / 2<m<1$. These properties are also in agreement with the results of the numerical calculations as it could be oberved in Figs. 5 and 6.

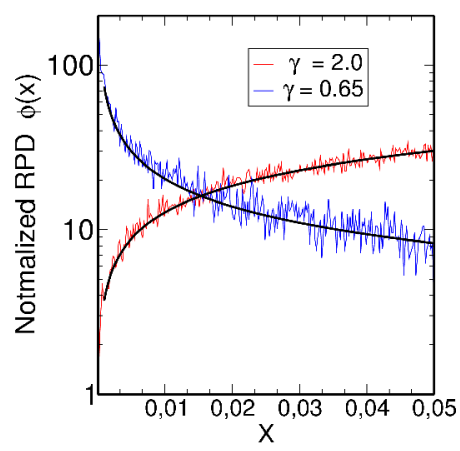

Figure 5. Comparison of $\phi(x)$ for the map (1) compared with Eq. (4) (solid line) using the numerical data of Fig. 3.

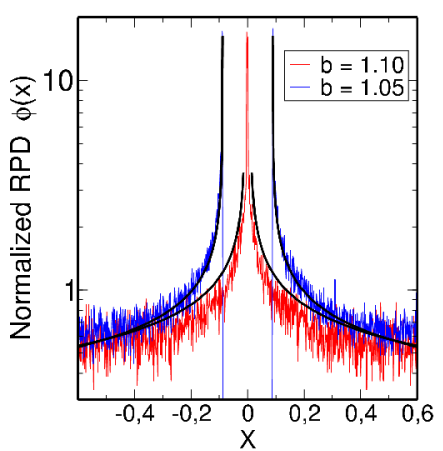

Figure 6. The same of Fig 5 for the map of Eq. (2) using the data of Fig. 4. The gap around the unstable point is evidenced.

The expression (3) filters the usual noise of the numerical data; and allows us to obtain a better description than the numerical reinjection density. It is important to emphasize that a LBR different from zero (see Figs 4 and 6) produces a gap around the unstable point in the Poincaré map. Such gap around the unstable point was observed in early experiments [7,2]. Its existence is justified by the linear dependence of $M(x)$ according to the values of $d$. In first place, when $d \approx 0$ and simultaneously we have $0<\alpha$, the function $\phi(x)$ approaches to zero as $x$ tends to the unstable point. In this case, the gap would be filled when we have in the Poincaré return map a large enough number of points. However, for a finite sampling $d>0$ and the aforementioned gap develops as shown in Fig 5.

\section{The effect of the noise on the RPD}

Most of investigations on the role of an external noise in the Pomeau intermittency introduce random perturbations in the local map around the unstable point, without considering its effect on the RPD. In this section we study the effect of a superposed random signal to the maps of Fig. 1 and 2 as,

$$
x_{n+1}=G\left(x_{n}\right)+C \zeta(t)
$$

where $-1<\zeta(t)<+1$ is an uncorrelated white noise with amplitude $C$.

For non uniform RPD important changes would be expected in this distribution. On the contrary, for the particular case of the uniform reinjection the RPD still would remain independent of $x$. In Figures 7 and 8 are represented the numerical evaluation of $M(x)$ for different noise amplitudes $C$ in Eq. (7). These results are calculated with the same parameters used for the lower and upper lines in Figs. 2 and 3. The solid 
Del Río, E. et al.

bullets corresponding to the noiseless case $(C=0)$ and its linear fit (solid line) are represented in Fig.7. These previous results are compared with those with an added $(C>0)$ white noise.

For low values of $x$ the function $M(x)$ approaches the line with slope $1 / 2$ (dashed lines) corresponding to the case of uniform RPD. This effect is caused by the noise induced spread of iterations for small of $x$. For different noise amplitudes, the results of Figs. 9 and 10 suggest that $\phi(x)$ increases as the slope of $M(x)$ grows. This fact suggests, a new noise induced reinjection process that. For the map of Eq. (1) this means there points with $x<x_{r}$ are iterated downwards, back into the laminar region. This would explain the upwards legs of Figs. 9 and 10 where two reinjection mechanisms coexist.

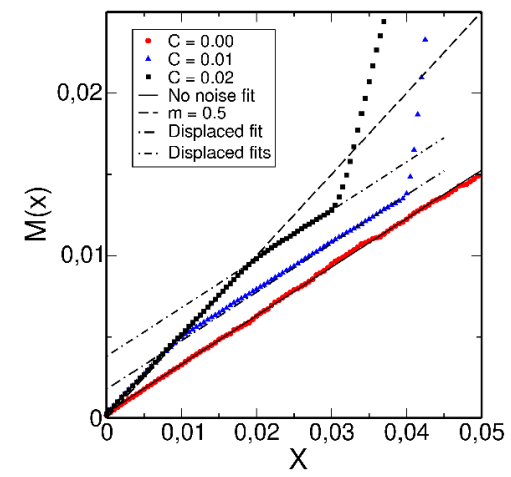

Figure 9. The numerical evaluation of $M(x)$ for the map (1) for different noise amplitudes in Eq. (8) for the same parameters as in Fig. 5.

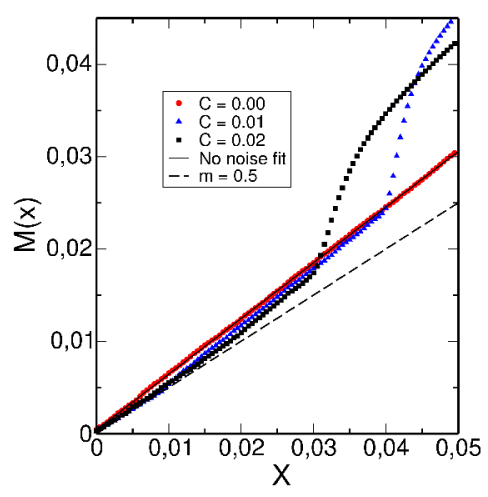

Figure 10. The numerical evaluation of $M(x)$ for the map (2) for different noise amplitudes in Eq. (8) for the same parameters as in Fig. 7.

\section{Conclusions and Discussion}

The function $M(x)$ constitutes a tool to evaluate the RPD and, in a number of cases, it is found that $M(x)=m\left(x-x_{i}\right)+d$, leading to

$\phi(x)=b\left(x-x_{i}\right)^{\alpha}$. Then the usual approximation of uniform reinjection approximation is recovered when $\alpha=0$ or equivalently $m=1 / 2$. Similar power dependence for the RPD have been observed in different maps which exhibit type II and III intermittencies. The existence of a minimum initial value $x_{r}$ for reinjection also provides an explanation for the gap around the unstable point experimentally reported [7]. It is currently assumed that the effect of the noise on the type-II and III intermittencies only affects the properties of the map locally around a stable point. On the contrary, our calculations using the function $M(x)$ indicate that the RPD is also strongly affected. 
Noise influence on the characteristic relations

The departure from the linear response observed in $M(x)$ in Figs. 9 and 10 evidence an increment of the RPD for larger values of $x_{r}$ and suggests the existence of a noise induced reinjection mechanism. The nonlinear expression for $M(x)$ and its relation between $\phi(x)$ and the noise amplitudes will be the subject of further investigations.

\section{Acknowledgements}

This research was supported by the Technical University of Madrid (UPM) under grant AL09-PID-03 and by CONICET under grant PID-5269, Universidad Nacional de Córdoba and Ministerio de Ciencia y Tecnología de Córdoba. Dr. J.M Donoso and Dr. L. Conde acknowledge the partial support of Spanish Ministry of Science and Innovation under Grant ENE2007-67406C02-01.

\section{References}

[1] E. Del Rio and S. Elaskar. New characteristic relations in type-II intermittency. Int. J. Bif. Chaos, 20. (4). pp. 1-7 (2010)

[2] E. Del Río, M.G. Velarde and A. Rodríguez-Lozano. Long time data series and difficulties with the characterization of chaotic attractors: a case study with intermittency III. Chaos. Sol. Frac., 4. (12). pp. 2169-2179 (1994)

[3] H.G. Schuster and W. Just. Deterministic Chaos: An Introduction. Wiley-VCH Verlag GmbH and Co. KGaA, Weinheim, Germany (2005)

[4] P. Manneville. Intermittency, self-similarity and $1 / \mathrm{f}$ spectrum in dissipative dynamical systems. J. Physique, 41. pp. 1235-1243 (1980)

[5] Y. Pomeau and P. Manneville. Intermittent transitions to turbulence in dissipative dynamical systems. Comm. Math. Phys., 74. pp. 89-197 (1980)

[6] F. R. C. Grebogi, E. Ott and J. Yorke. Critical exponents for crisis induced intermittency. Phys. Rev. A, 36. pp. 5365-5380 (1987)

[7] M.A. Rubio M. Dubois and P. Bergé. Experimental evidence of intermittencies associated with a subharmonic bifurcation. Phys. Rev. Lett. 51. pp. 1446-1449 (1983)

[8] Lee Eok-Kyun Kim Chil-Min, O.J. Kwon and Lee Hoyun. New characteristic relations in Type-I intermittency. Phys. Rev. Lett. 73, pp. 525-528 (1994)

[9] Kim Yeon-Soo Kim Jeong-Moog Kim Chil-Min, Yim Geo-Su and H.W. Lee. Experimental evidence of characteristic relations of type-I intermittency in an electronic circuit. Phys. Rev. E, 56. pp. 2573-2577 (1997) 
Del Río, E. et al.

[10] Ryu Jung-Wan Kim Chil-Min, Yim Geo-Su and Park Young-Jai. Characteristic relations of type-III intermittency in an electronic circuit. Phys. Rev. Lett. 80. pp. 5317-5320 (1998)

[11] J.J. Zebrowski and R. Baranowski. Type-I intermittency in nonstationary systems: models and human heart-rate variability. Physica A. 336. pp. 74-83 (2004)

[12] J.P. Farmer D. Crutchfield and B.A. Huberman. Fluctuations and simple chaotic dynamics. Phys. Rep. 92. pp. 45-82 (1982)

[13] A.S. Pikovsky. A new type of intermittent transition to chaos. J. Phys. A. 16. L109-112 (1983)

[14] Park Young-Jai Cho Jin-Hang, Ko Myung-Suk and Kim Chil-Min. Experimental observation of the characteristic relations of type-I intermittency in the presence of noise. Phys. Rev. E. 65. 036222 (2002)

[15] Kye Won-Ho and Kim Chil-Min. Characteristic relations of type-I intermittency in the presence of noise. Phys.Rev. E. 62. pp. 6304-6307 (2000)

[16] Rim Sunghwan Kye Won-Ho and Kim Chil-Min. Experimental observation of characteristic relations of type-III intermittency in the presence of noise in a simple electronic circuit. Phys.Rev. E. 68. 036203 (2003)

[17] S. Elaskar, E. Del Rio and J. Donoso. Reinjection probability density in type-III intermittency. Int. J. Bif. Chaos, Submitted. 